\title{
Avaliação da resistência de leveduras biocontroladoras à substâncias "GRAS" - (Generally Regarded As Safe)
}

\begin{abstract}
Eskálath Morganna Silva FERREIRA ${ }^{[1 ; *]}$; Camilla Martins MALTA ${ }^{[1]}$; Leisciany Vieira GONZAGA ${ }^{[1]}$, Jordana Oliveira BICALHO ${ }^{[1]}$, Raphael Sanzio PIMENTA ${ }^{[2]}$

${ }^{[1]}$ Universidade Federal do Tocantins, Campus Palmas. Laboratório de Microbiologia Geral e Aplicada. Avenida NS 15, 109 Norte - Plano Diretor Norte, 77001-090. Palmas - TO, Brasil. Email: camillamalta_17@hotmail.com, leiscyma@gmail.com, jordana.eng.amb@gmail.com

${ }^{[2]}$ Professor do programa de Pós-Graduação Doutorado em Biodiversidade e Biotecnologia da Amazônia Legal (BIONORTE). Laboratório de Microbiologia geral e Aplicada. Avenida NS 15, 109 Norte - Plano Diretor Norte, 77001-090. Palmas-TO, Brasil. E-mail: biorapha@yahoo.com.br

INFORMAÇÕES

Recebido em: 06/09/2015

Aceito em: 25/11/2015

Publicado em: 23/12/2015

\begin{tabular}{l}
\hline Document Object Identifier \\
$10.18067 / j b f s . v 2 \mathrm{i} 4.66$ \\
\hline
\end{tabular}

Termos de indexação:

Controle biológico integrado

Biocontrole

Bicarbonato de sódio

*Autor para correspondência

morganna_ferreira@hotmail.com

O controle biológico tem sido integrado ao uso de substâncias conhecidas como "GRAS" (Generally Regarded As Safe), estas substâncias são normalmente utilizadas como aditivos alimentícios, e seu principal papel é auxiliar os agentes biológicos em sua ação antagônica, potencializando assim o controle biológico. O presente trabalho teve como objetivo observar os efeitos de cinco substâncias "GRAS" no crescimento de quatro isolados de leveduras (5822, 5852, 5859, 5872) selecionados por apresentarem características de bons agentes biocontroladores. Para realização dos testes, os isolados foram crescidos em caldo GYMP acrescido das substâncias bicarbonato de sódio, carbonato de sódio, carbonato de cálcio, cloreto de cálcio e cloreto de potássio nas concentrações de $1 \%, 3 \%$ e $5 \%$ com exceção do cloreto de potássio cujas concentrações foram de $0,1 \%, 0,5 \%$ e $1 \%$ (concentração máxima permitida pela legislação). Nos dias 1, 4, 7, e 10 de incubação uma alíquota de $50 \mu 1$ foi retirada das culturas e plaqueadas, sendo os resultados expressos em UFC/mL. Entre os quatro isolados testados, somente o 5852 foi capaz de resistir por sete dias na presença da substância bicarbonato de sódio, nas três concentrações testadas. Demonstrando a capacidade desta levedura em ser utilizada em protocolos de controle biológico integrado a esta substância
\end{abstract}

\section{Resistance evaluation biological controller yeast to substances "GRAS" - (Generally Regarded As Safe)}

ABSTRACT- Biological control has been integrated into substances known as "GRAS" (Generally Regarded As Safe), these substances are commonly used as food additives, and its main role is to assist the biological agents antagonistic action. This study aimed to test the effects of five "GRAS substances " over the growth of four strains of yeast $(5822,5852,5859,5872)$ selected by presenting characteristics of good biocontrol agents. To perform the test, the strains were grown in GYMP broth with the addition of substances sodium bicarbonate, sodium carbonate, calcium carbonate, calcium chloride and potassium chloride at concentrations of $1 \%, 3 \%$ and $5 \%$ with the exception of chloride whose potassium concentrations were $0.1 \%, 0.5 \%$ and $1 \%$ (maximum concentration permitted by law). On days 1, 4, 7, and 10 after incubation an aliquot of $50 \mu 1$ was removed and plated cultures, and the results expressed as CFU / $\mathrm{ml}$. Among the four tested strains, only 5852 was able to survive seven days in the presence of sodium bicarbonate at the three concentrations tested. Demonstrating the ability of this yeast to be used in integrated biological control protocols with this substance.

Index terms: integrated biological control; Biocontrol; Sodium bicarbonate.

c) (i) (2)(-) Copyright: (C) 2015 JBFS all rights. This is an open-access article distributed under the terms of the Creative $\circlearrowleft$ EY No SA Commons Attribution License, which permits unrestricted use, distribution, and reproduction in any medium, provided the original author and source are credited.

Financiamento: Universidade Federal do Tocantins e CNPq.

Conflito de interesse: Os autores declaram que não há conflito de interesse.

Como referir esse documento (ABNT):

FERREIRA, E. M. S.; MALTA, C. M.; GONZAGA, L. V.; BICALHO, J. O.; PIMENTA, R. S. Avaliação da resistência de leveduras biocontroladoras à substâncias "GRAS" - (Generally Regarded As Safe). Journal of Bioenergy and Food Science, Macapá, v.2, n.4, p.178-182, out./dez., 2015. http://dx.doi.org/10.18067/jbfs.v2i4.66 


\section{INTRODUÇÃO}

O conhecimento acerca de microrganismos como agente de controle biológico, remota a centenas de anos. Porém, o interesse por microrganismos antagonistas, principalmente as leveduras, tem crescido ao longo das duas últimas décadas, devido estudos apontarem resultados significativamente positivos de controle [1-6].

As leveduras são particularmente os microrganismos mais adequados para utilização como agente de controle biológico devido à sua alta capacidade de colonizar superfícies vegetais e manterem-se viáveis durante longos períodos de tempo sob diferentes condições ambientais [7, 8].

A utilização de leveduras antagonista associadas a uma substância GRAS (Generally Regarded As Safe), ou seja, uma substância considerada segura para consumo caracteriza o controle biológico integrado e tem se mostrado uma alternativa ainda mais eficiente que o controle biológico clássico, ao qual se utiliza apenas o micro-organismo antagonista contra o patógeno alvo de controle. Em geral, quando se realiza a combinação entre tratamentos deseja se obter efeitos aditivos ou sinérgicos para aumentar a eficácia ou a persistência dos tratamentos individuais, assim como aumentar os efeitos complementares combinando atividades preventivas [8].

O controle biológico integrado é uma estratégia que têm aumentado a atenção de pesquisadores, pois ocorre a consorciação dos dois tipos de tratamentos, a fim de superar o desempenho e aumentar a variável de eficácia das abordagens alternativas existentes [9].

Como demonstra o estudo realizado por Gamagae et al., [10], ao utilizarem o bicarbonato de sódio a $2 \%$ associado a Candida oleophila no controle da antracnose em mamão, onde observaram que houve um aumento no controle da doença. Chen et al. [1], também constataram que a eficiência do controle biológico foi maior com a adição de $2 \%$ de bicarbonato de sódio em conjunto com a levedura Kluyveromyces marxianus contra Penicillium digitatum em citrus. Da mesma forma, Pimenta et al. [11] observaram em seus estudos que ao utilizar a levedura Saccharomycopsis crataegensis em consorciação com bicarboanto de sódio a 5\% houve controle total do Penicillium digitatum em laranjas.

Assim as substâncias "GRAS" mostram-se então como uma boa alternativa no controle de fitopatógenos de frutas e plantas, pois auxiliam outros agentes biológicos em sua ação antagônica, podendo causar efeitos fungistático ou fungicida ao patógeno alvo de controle. Estas são substâncias sancionadas pela FAO/FDA já utilizadas como aditivos alimentícios, portanto, não causam danos à saúde e não necessitam de avaliação prévia de uso e comercialização, o que facilita ainda mais a sua utilização.

Diante do exposto, o presente trabalho teve como finalidade observar a resistência de quatro isolados de leveduras $(5822,5852,5859,5872)$ selecionados por apresentarem características de agentes de controle biológico a cinco substâncias "GRAS". Verificando desta forma a possibilidade de utilização destas substâncias, integradas aos agentes previamente selecionados.

\section{MATERIAL E MÉTODOS}

\section{a) Obtenção dos isolados}

Os isolados foram obtidos a partir da coleção de cultura Carlos Henrique Rosa do Laboratório de Microbiologia Geral e Aplicada da Universidade Federal do Tocantins do Tocantins. Estes isolados foram selecionados para o estudo devido apresentarem perfil de agentes de controle biológico verificados em estudos anteriores (dados não mostrados).

\section{b) Teste de Resistência a substâncias "GRAS"}

Para o estudo foram selecionadas cinco substâncias listadas pela Food and Drug Administration-FDA [12] sendo elas: bicarbonato de sódio, carbonato de sódio, carbonato de cálcio, cloreto de cálcio e cloreto de potássio. Estas substâncias foram empregadas no estudo pelo fato de estudos, já relatarem seus possíveis efeitos fungistáticos e fungicidas a fitopatógenos de plantas [13].

Para o estudo os isolados foram crescidos inicialmente para obtenção de biomassa em caldo GYMP (1\% extrato de levedura; $0,5 \%$ Peptona; $1 \%$ glicose; $0,02 \%$ fosfato de sódio monobásico) e incubadas a $25 \pm 2{ }^{\circ} \mathrm{C}$ por $48 \mathrm{~h}$ a $100 \mathrm{rpm}$. Utilizou-se câmara de Neubauer para contagem de células e a seguir a concentração foi ajustada $1 \times 10^{6}$ células $/ \mathrm{mL}$.

Após a obtenção da concentração inicial, foram realizadas inoculações em caldo GYMP acrescido das substâncias citadas nas concentrações de $1 \%, 3 \%$ e $5 \%$ com exceção do cloreto de potássio cuja concentração foi de $0,1 \%, 0,5 \%$ e $1 \%$. Os erlenmeyers foram então incubadas a $25 \pm 2{ }^{\circ} \mathrm{C}$ por até 10 dias. Durante os dias 1, 4, 7, e 10 uma alíquota de $50 \mu \mathrm{l}$ foi retirada das culturas incubadas e plaqueadas utilizando o equipamento Eddy Jet Spiral plater (IUL, INSTRUMENTS) em placas de 
Petri contendo meio NYDA $(0,3 \%$ extrato de carne; $0,5 \%$ extrato de levedura; $0,5 \%$ peptona; $1 \%$ glicose; $2 \%$ ágar; $0,02 \%$ cloranfenicol) e incubadas a temperatura de $25 \pm 2{ }^{\circ} \mathrm{C}$ por $48 \mathrm{~h}$.

A leitura foi realizada pelo equipamento Flash \& gol (IUL, INSTRUMENTS) e os resultados foram expressos em UFC/mL (Unidades Formadoras de Colônias).

\section{RESULTADOS E DISCUSSÃO}

Entre os quatro isolados testados, apenas o 5852 foi capaz de resistir por até sete dias na presença de pelo menos uma das substâncias GRAS testada (bicarbonato de sódio). Os outros isolados não foram capazes de resistir a nenhuma das substâncias utilizadas no estudo e em nenhuma concentração avaliada. Isso demonstra uma alta sensibilidade que estes isolados possuem as substâncias testadas e não compatibilidade de utilização destas substâncias ao controle biológico integrado com estas leveduras.

A sobrevivência do isolado $5852 \mathrm{em}$ formulações com 1, 3, e 5\% de bicarbonato sódio in vitro pode ser considerada satisfatória, uma vez que a levedura permaneceu viável em todas estas concentrações após uma semana de incubação (Figura 1). A população do isolado somente foi reduzida após 4 dias, e durante os dias 4 e 7 de incubação a análise estatística verificou que não houveram diferenças ao nível de $5 \%$ de significância pelo teste de Tukey entre as três concentrações testadas em relação ao controle (Tabela 1).

Figura 1. Efeito de diferentes concentrações de bicarbonato de sódio (BS) no crescimento do isolado 5852 in vitro.

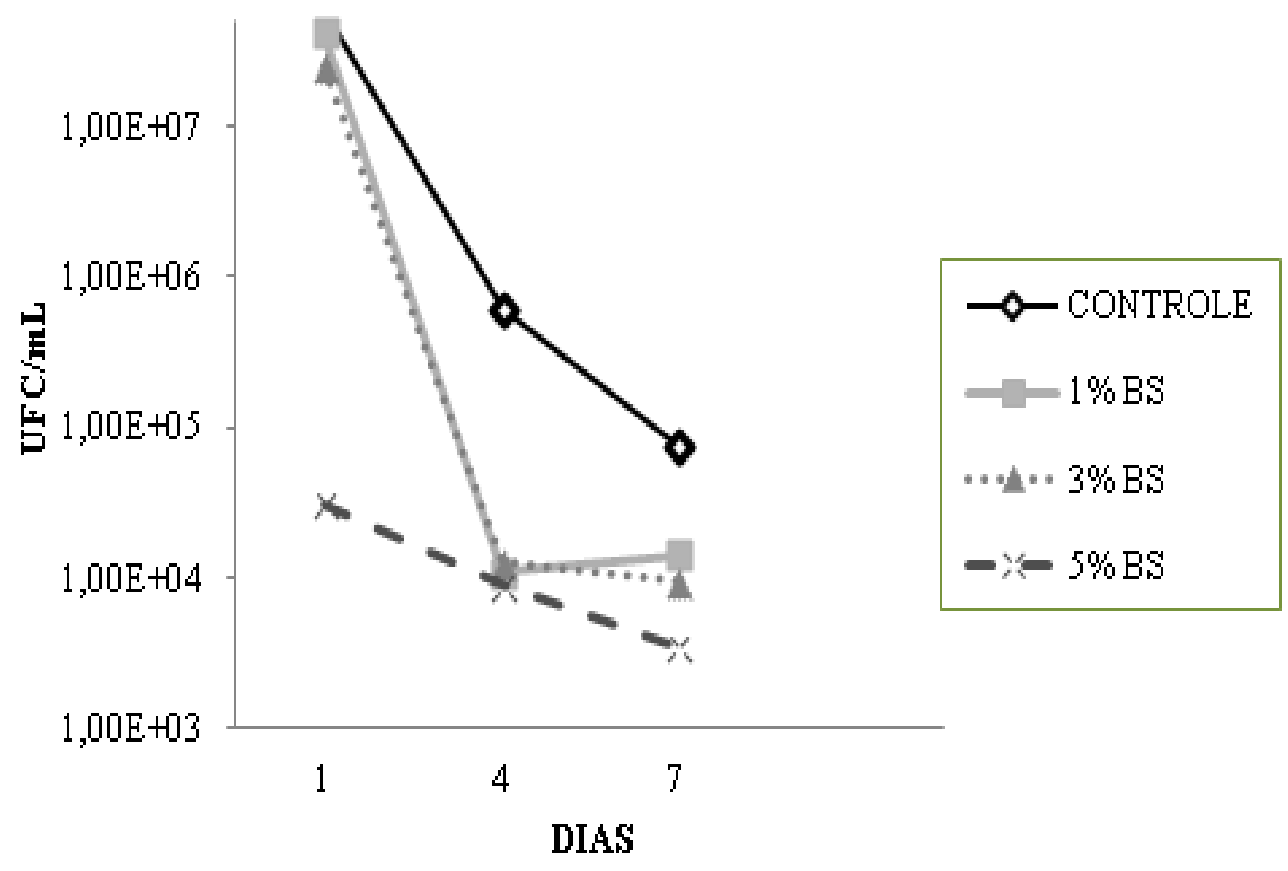

Tabela 1. Médias de crescimento do isolado 5852 em Unidades formadoras de colônias por $\mathrm{mL}$ (UFC/mL) durante sete dias de incubação.

\begin{tabular}{crcc}
\hline TRATAMENTOS & $1^{\circ}$ & $4^{\circ}$ & $7^{\circ}$ \\
\hline Controle & $5,7 \times 10^{7} \mathrm{a}$ & $5,9 \times 10^{5} \mathrm{a}$ & $7,5 \times 10^{4} \mathrm{a}$ \\
$1 \%$ Bic. Sódio & $4,1 \times 10^{7} \mathrm{a}$ & $1,1 \times 10^{4} \mathrm{a}$ & $1,4 \times 10^{4} \mathrm{a}$ \\
$3 \%$ Bic. Sódio & $2,4 \times 10^{7} \mathrm{ab}$ & $1,2 \times 10^{4} \mathrm{a}$ & $9,7 \times 10^{3} \mathrm{a}$ \\
$5 \%$ Bic. Sódio & $3,1 \times 10^{4} \mathrm{~b}$ & $9,7 \times 10^{3} \mathrm{a}$ & $3,4 \times 10^{3} \mathrm{a}$ \\
\hline
\end{tabular}

*Médias seguidas de letras minúsculas distintas nas colunas diferem entre si pelo teste de Tukey $(\mathrm{p} \leq 0,05)$. 
Normalmente a combinação de substâncias GRAS com agentes de controle biológico tem exibido um efeito sinérgico, tendo a maioria dos estudos comprovado a possibilidade da consorciação do uso de leveduras com várias destas substâncias, principalmente o bicarbonato de sódio $[10,11,14]$.

Conforme Pimenta et al. [11] a forte resistência ao bicarbonato de sódio é uma característica importante a leveduras, isso permite o uso destas substâncias em concentrações mais elevadas, tendo melhor efeito na redução da deterioração de frutas.

Entretanto, a concentração de bicarbonato de sódio a $1 \%$ apresentou-se como significativa não diferindo estatisticamente das concentrações mais elevadas na inibição do patógeno durante os testes in vitro. Isto demonstra boa eficiência de controle e preservação do antagonista na menor concentração testada. O que é de grande importância, pois o uso de concentrações mais baixas pode promover a redução dos custos de aplicação do controle biológico integrado com a mesma eficiência.

\section{CONCLUSÃO}

Entre os quatro isolados testados, somente o 5852 foi capaz de resistir por sete dias na presença da substância bicarbonato de sódio, nas três concentrações testadas.

Sendo assim o isolado 5852 demonstrou capacidade em ser utilizado em protocolos de controle biológico integrado a substancia bicarbonato de sódio

\section{CONTRIBUIÇÃO DOS AUTORES}

1. Condução do experimento, análises e elaboração do artigo:

Eskálath Morganna Silva Ferreira

Camilla Martins Malta

Leisciany Vieira Gonzaga

Jordana Oliveira Bicalho

2. Planejamento, orientação e revisão final do artigo:

Dr. Raphael Sanzio Pimenta

Eskálath Morganna Silva Ferreira

\section{REFERÊNCIAS}

[1]. CHEN, P.S.; HU,M.; UL-MAQ, M.R.; LAI, K.; QU, F.; ZHANG, Y. Combination of Kluyveromyces marxianus and sodium bicarbonate for controlling green mold of citrus fruit. International Journal of Food Microbiology. v.151, p.190-194, 2011. DOI:10.1016/j.ijfoodmicro.2011.08.023

[2]. JANISIEWICZ, W. J.; CONWAY, W.S. Combining biological control with physical and chemical treatments to control fruit decay after harvest. An international journal for reviews in postharvest biology and technology. 2010.

[3]. LU, H.; LU, L.; ZENG, L.; FU,D.; XIANG,H.; YU,T.; ZHENG, X. Effect of chitin on the antagonistic activity of Rhodosporidium paludigenum against Penicillium expansum in apple fruit. Postharvest Biology and Technology, v.92, p.9-15, 2014.

[4]. MARI, M.; MARTINI, C.; GUIDARELLI, M.; NERI, F. Postharvest biocontrol of Monilinia laxa, Monilinia fructicola and Monilinia fructigena on stone fruit by two Aureobasidium pullulansstrains. Biological Control, v.60, p.132-140, 2012. DOI: 10.1016/j.biocontrol.2011.10.013

[5]. NALLY, M.C.; PESCE, V.M.; MATURANO, Y.P.; MUÑOZ, C.J.; COMBINA, M.; TORO, M.E.; CASTELLO, L.I.; VALQUEZ, F. Biocontrol of Botrytis cinérea in table grapes by non-pathogenic indigenous Saccharomyces cerevisiae yeasts isolated from viticultural environments in Argentina. Postharvest Biology and Technology, v.64, p.4044, 2012. DOI:10.1016/j.postharvbio.2011.09.009

[6]. TRISTÃO, G.B.; MALTA, C.M.; BORGES, A.K.P.; MORAIS, P.B.; SILVA, J.F.M.; PIMENTA, R.S. Leveduras Associadas A Frutos De Abacaxi (Ananas Comosus) e Sua Utilização Como Agentes De Controle Biológico, Engenharia ambiental v.9, n.2, p.085-093, 2012.

[7]. PALOU, L., SMILANICK, J.L. AND DROBY, S. Alternatives toconventional fungicides for the control of citruspostharvest green and blue moulds. Stewart Posthar Review. v.4, p.1-16, 2008. DOI:10.2212/spr.2008.2.2

[8]. PIMENTA, R.S.; MORAIS, P.B.; ROSA, C.A.; CORRÊA, J. Utilization of Yeasts in Biological Control Programs, Yeast Biotechnology: Diversity and Applications. SPRINGER SCIENCE, 2009. 200-212p

[9]. PIMENTA, R.S.; SILVA, J.F.M.; BUYER, J.S.; JANISIEWICZ, W.J. Endophytic Fungi from Plums (Prunus domestica) and their Antifungal Activity against Monilinia fructicola. Journal of Food Protection, v. 75, p. 1883-1889, 2012.

[10]. GAMAGAE, S. U.; SIVAKUMAR, A.D.; WILSON WIJERATNAM R.S.; WIJESUNDERA, R.L.C. Use of sodium bicarbonate and Candida 
oleophila to controlanthracnose in papaya during storage. Crop Protection, v. 22, p.775-779, 2003.

[11]. PIMENTA, R.S.; SILVA, J. F.M.; COELHO, C.M.; MORAIS, P. B. Integrated control of Penicillium digitatum by the predacious yeast Saccharomycopsis crataegensis and sodium bicarbonate on oranges. Brazilian Journal of Microbiology. v.41, p.404-410, 2010. DOI:10.1590/S1517-83822010000200022

[12]. FDA - FOOD SAFETY AND INSPECTION SERVICE. Federal Register. Rules and Regulations. v. 64, n. 246, 1999.
[13]. JANISIEWICZ, W. J; KURTZMAN, C. P.; BUYER, J. S. Yeasts associated with nectarines and their potential for biological control of brown rot. Yeast. v.27, p. 389-398, 2010. DOI:10.1002/yea.1763

[14]. GENG, P.; CHEN, S.; HU, M.; RIZWAN-ULHAQ, M.; FEI QU,K.L; ZHANG, L. Combination of Kluyveromyces marxianus and sodium bicarbonate for controlling green mold of citrus fruit. International Journal of Food Microbiology, v. 151 , p.190-194, 2011. 